\title{
Potential of afatinib in the treatment of patients with HER2-positive breast cancer
}

This article was published in the following Dove Press journal:

Breast Cancer:Targets and Therapy

24 August 2012

Number of times this article has been viewed

\author{
Elena Geuna' \\ Filippo Montemurro ${ }^{2-4}$ \\ Massimo Aglietta ${ }^{1-3,5}$ \\ Giorgio Valabrega ${ }^{1-3,5}$ \\ 'Division of Medical Oncology, \\ Institute for Cancer Research and \\ Treatment, Candiolo, Turin, ${ }^{2}$ Institute \\ for Cancer Research, Candiolo, Turin, \\ ${ }^{3}$ Foundation of Piedmont Oncology, \\ Candiolo, Turin, ${ }^{4}$ Unit of Investigative \\ Clinical Oncology, Candiolo, Turin, \\ ${ }^{5}$ University Medical School of Turin, \\ Turin, Italy
}

\begin{abstract}
In the absence of treatment, overexpression of the human epidermal growth factor receptor 2 (HER2) predicts a poor prognosis in breast cancer. In the last decade, monoclonal antibodies and small molecule tyrosine kinase inhibitors have significantly improved the outcome of HER2-positive breast cancer patients. However, tumor resistance and toxicities often limit the use of these therapies. For this reason, there is a compelling need for further investigation of new targeted therapies, such as afatinib, an oral irreversible pan inhibitor of the epidermal growth factor receptor (EGFR) family. This compound covalently interacts with tyrosine kinase domains, which are deeply involved in signal transduction leading to cell proliferation and protection from apoptosis. Afatinib has been studied in several Phase I clinical trials in advanced solid tumors. These trials have shown encouraging clinical activity and manageable side effects when afatinib is used either as a single agent or in combination with chemotherapy, with cutaneous adverse events and diarrhea being the most frequently observed toxicities. This review will focus on afatinib's clinical activity and will discuss ongoing clinical studies in HER2-positive breast cancer patients. In the scenario of the different HER2-targeted therapies, it will be important to define the best specific clinical and "molecular" setting for afatinib use, trying to identify predictors of resistance and response. Moreover, afatinib, which has the ability to cross the blood-brain barrier, could play a role in patients with brain metastases from breast cancer.
\end{abstract}

Keywords: afatinib, brain metastasis, human epidermal growth factor receptor 2, metastatic breast cancer, monoclonal antibodies, small molecule kinase inhibitors

\section{Introduction}

The epidermal growth factor receptor (EGFR) family represents a complex biological network. Each of the four members form both homo- and heterodimers within the EGFRfamily and with other biological systems such as insulin-like growth factor 1 receptor, the estrogen receptor (ER), and the angiogenic pathway. EGFR family members can react to and circumvent different perturbations and blockades induced by targeted therapies, maintaining therefore potent proliferative and antiapoptotic intracellular signals. These adaptive mechanisms contribute significantly to the phenomenon of drug resistance. Emerging clinical data indicate that, although progressing on HER2 inhibitors. Breast cancer can persistently depend on HER2-dependent signaling pathways HER2dependent signaling pathways and, consequently a continuous HER2 inhibition remains the backbone of treatment in patients through different lines of treatment ${ }^{1,2}$ (Figure 1). For example, there is evidence that other anti-HER2 therapies (such as lapatinib, the monoclonal antibody pertuzumab, the antibody-drug conjugate trastuzumab DM1,
Correspondence: Giorgio Valabrega Institute for Cancer Research and Treatment, Strada Provinciale 142, km 3.9510060 Candiolo, Torino, Italy $\mathrm{Tel}+390119933253$

Fax +390119933275

Email giorgio.valabrega@ircc.it 


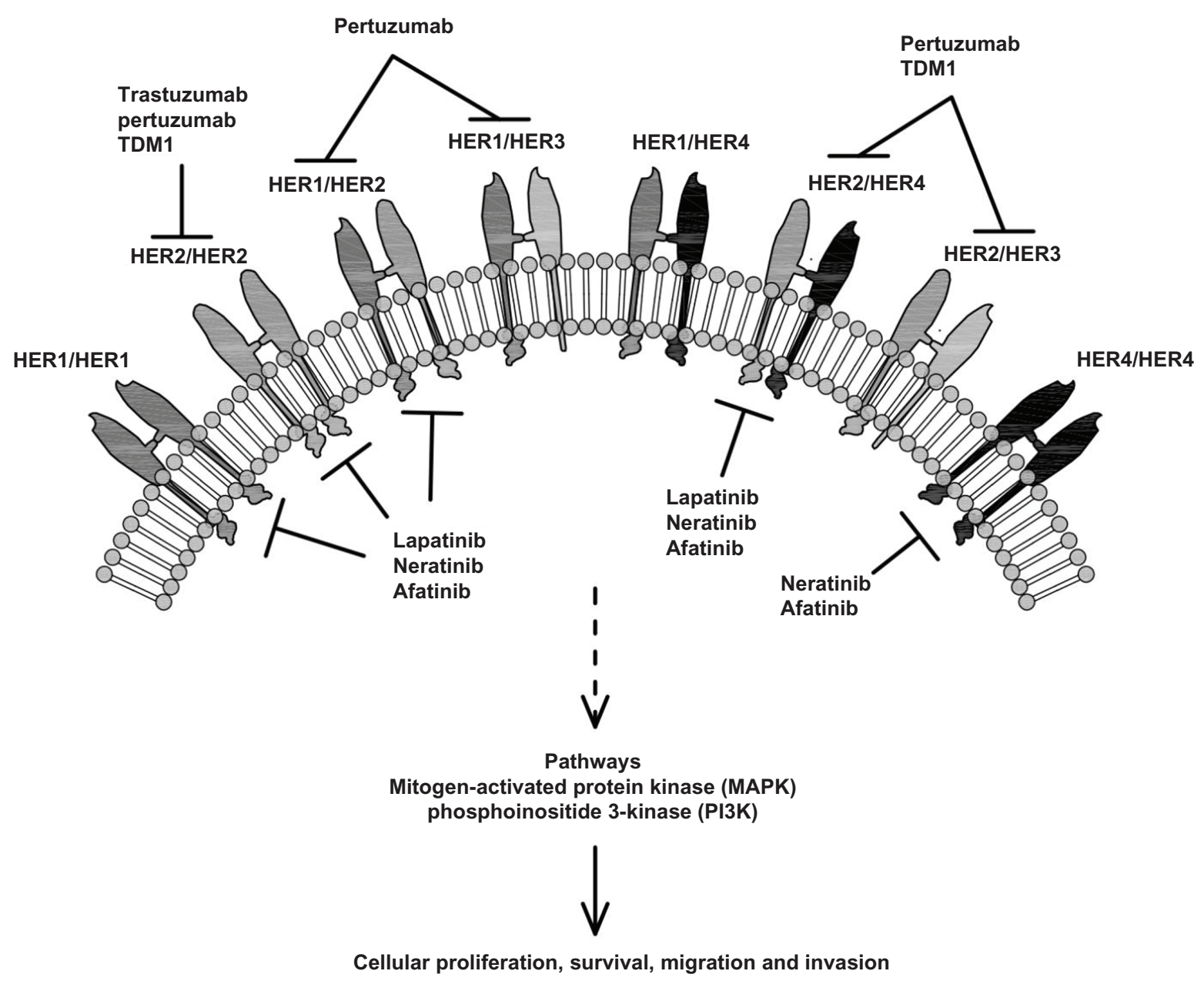

Figure I HER family receptors and some therapeutic agents available or in development.

Notes: HER2 forms homo- and heterodimers with other members of the EGFR family (HERI, HER3 and HER4). Dimerization activates multiple downstream signaling cascades including the mitogen-activated protein kinase (MAPK) and phosphoinositide 3-kinase (PI3K) pathways which promote cellular proliferation, survival, migration and invasion. Trastuzumab represents the first milestone of drugs targeting HER2 as individual receptor. Dual-targeting agents include monoclonal antibodies (Pertuzumab and TDM-I) and small molecule tyrosine kinase inhibitors (Lapatinib, Neratinib, Afatinib).

the oral irreversible dual EGFR inhibitor neratinib but also the combination trastuzumab-lapatinib) are still active beyond progression on a first-line anti-HER2 treatment. ${ }^{3-7}$

These reasons outline the compelling need for the investigation and development of new anti-HER2-targeted therapies and compounds that could be used for continuous HER2 inhibition.

This review will focus on afatinib, another oral irreversible multitarget inhibitor of the EGFR family, and on its activity in HER2-positive breast cancer patients.

In other malignancies, such as lung cancer, afatinib has an emerging role in overcoming resistance to reversible EGFR tyrosine kinase inhibitors (TKIs) with an encouraging antitumoral activity, especially in non-small-cell lung cancer (NSCLC) harboring EGFR/HER1-activating mutations. Consequently, afatinib in patients with NSCLCresistant to reversible TKIs is being explored in a number of Phase I and II clinical trials. ${ }^{8-10}$ At the 2012 American Society of Clinical Oncology (ASCO) meeting, results from a Phase III study of afatinib versus pemetrexed-cisplatin as first-line in advanced adenocarcinoma of the lung (LUXLung 3 ) found that treatment with afatinib increases response rate and significantly prolongs progression-free survival. ${ }^{11}$

However, the role of afatinib is much more undefined in breast cancer.

\section{Afatinib: mechanism of action and preclinical activity}

Afatinib (BIBW 2992) is a novel, orally bioavailable, anilinoquinazoline compound, developed by Boehringer Ingelheim Pharma GmbH, (Ingelheim, Germany). This agent acts as a potent, irreversible, highly selective inhibitor of EGFR/HER1, HER2, and HER4 tyrosine kinase activity (Figure 1). It covalently interacts with cysteine 773 of EGFR 
and cysteine 805 of HER2 adenosine triphosphate (ATP)binding sites, with half-maximal inhibitory concentration values of 0.5 and $14 \mathrm{nM}$, respectively. ${ }^{8}$ Furthermore, afatinib inhibits EGFR/HER1- and HER2-containing dimers. ${ }^{8,12}$ As these receptors are deeply involved in cell proliferation and apoptosis inhibition, their suppression may play a critical role in tumor growth control (Figure 1).

Afatinib has demonstrated preclinical activity in a number of different preclinical tumor models, including EGFR- and HER2-overexpressing trastuzumab-resistant cell lines (SUM 190-PT) as well as HER2-negative cell lines (SUM 149-PT) and in several in vivo models. ${ }^{8,10}$ In particular, afatinib showed activity in mouse xenografts overexpressing EGFR/ HER1 and/or HER2, inducing partial and complete tumor shrinkage. ${ }^{12}$

Moreover, due to its covalent binding to the tyrosine kinase active site, afatinib showed an efficient inhibitory activity in a variety of EGFR mutants including the acquired second-site resistance mutation T790M and wild-type and mutant HER2, which is well known for being resistant to first-generation EGFR TKIs. ${ }^{8}$

\section{Phase I trials}

Afatinib has been investigated in multiple Phase I clinical trials. ${ }^{8,13,14}$ These studies have revealed a manageable side effect profile both when used as a single agent and in combination with chemotherapeutic drugs (including cisplatin/paclitaxel, cisplatin/5-fluorouracil, and docetaxel). ${ }^{15-18}$ The first in-human study evaluated an escalating schedule of once-daily afatinib for 14 days followed by 14 days off treatment in patients with advanced solid tumors. ${ }^{13}$ On the basis of this study, the recommended Phase II dose in this schedule (14 days on and 14 days off) has been established between 50 and $70 \mathrm{mg}$ once daily. Observed adverse events (AEs), which are always reversible, consisted of cutaneous (rash, acne, and dry skin) and gastrointestinal toxicities (diarrhea, mucositis, nausea, and vomiting), which usually resulted in dose limiting. Differently from other anti-HER2 target therapies, afatinib is not associated with significant left ventricular ejection fraction (LVEF) changes. ${ }^{19,20}$ Its pharmacokinetic profile revealed oral bioavailability, moderately fast absorption, and a half-life ranging between 28 and 43 hours. Afatinib is rapidly distributed in the tissues with moderate-to-high clearance. The major route of elimination of afatinib was via the feces. Pharmacodynamic analyses in skin biopsies did not show remarkable changes in EGFR-associated biomarkers; however, there was a significant reduction in epidermal keratinocytes proliferation scored with Ki67 index. Neither complete nor partial responses were observed. Disease stability lasting more than four cycles was seen in seven patients with different tumor types (especially colorectal and NSCLC). ${ }^{13}$

Other studies have explored different schedules of administration of afatinib. In all these studies, afatinib was well tolerated with similar AEs reported. ${ }^{19-21}$ Afatinib was escalated up to $100 \mathrm{mg}$ daily on an intermittent schedule with an increased frequency and severity of drug-related AEs for doses higher than 50-70 mg daily. A trial evaluating daily continuous dosing schedule of afatinib was reported by Yap and colleagues. ${ }^{14}$ Fifty-three patients were enrolled in this study and assigned to five different dose levels from 10 to $50 \mathrm{mg}$ daily continuously administered, with a determined maximumtolerated dose (MTD) of $50 \mathrm{mg}$ daily. The tolerability of afatinib was confirmed with diarrhea, nausea, vomiting, and rash as the most common AEs. These events were generally self-limiting or effectively controlled with specific medications. No cardiac toxicity was observed in terms of significant decline in ejection fraction. Dose limiting toxicities included grade 3 rash and reversible dyspnea secondary to pneumonitis. Differently from the previous described study, in Yap's trial three NSCLC patients had confirmed partial responses (two with an EGFR mutation), and disease stability lasting at least 6 months was reported in seven patients affected by colorectal, breast, cervical, and thyroid cancer.

\section{Activity in breast cancer}

A few Phase II clinical trials have tested afatinib in monotherapy or in combination (Table 1). Based on the Phase I study results, a $50 \mathrm{mg}$ daily dose was selected for Phase II studies. Hickish and colleagues conducted an openlabel Phase II study in which 41 patients with stage IIIB or IV HER2-positive breast cancer who had progressed on or were intolerant to trastuzumab were treated with singleagent afatinib at the dose of $50 \mathrm{mg} /$ day. ${ }^{22}$ Preliminary data demonstrated partial responses in four patients $(11 \%)$, one of whom remained on treatment until disease progression at 63 weeks, and 15 patients had stable disease for more than four cycles (37\%). Median progression-free survival was about 15 weeks while median overall survival was 61 weeks. ${ }^{23}$ The most common AEs were grade 3 diarrhea (24\%) and grade 3 skin rash (10\%), stomatitis and vomiting (7\%), and 17 patients required dose reductions due to AEs. There have been no grade 4 drug-related toxicities and no treatmentrelated deaths. This trial provided a rationale for further evaluation of afatinib in a larger cohort of HER2-positive, trastuzumab-resistant metastatic breast cancer patients. 
Table I Afatinib activity in breast cancer: main studies

\begin{tabular}{|c|c|c|c|}
\hline Authors & $\mathbf{N}^{\circ}$ patients & Study design & Key clinical results \\
\hline Hickish et $\mathrm{al}^{22}$ & 41 & $\begin{array}{l}\text { Phase 2: patients with stage IIlb or IV breast cancer } \\
\text { progressed on trastuzumab treated with } \\
\text { afatinib } 50 \mathrm{mg} / \text { day monotherapy }\end{array}$ & $\begin{array}{l}\text { II\% PR; } 43 \% \text { SD } \\
\text { AE: diarrhea (24\%) and } \\
\text { skin rash (I0\%) G3 }\end{array}$ \\
\hline Harbeck et $\mathrm{al}^{24}$ & 56 & $\begin{array}{l}\text { Phase 2: patients with HER2 negative hormone receptor } \\
\text { positive or triple negative, metastatic breast cancer } \\
\text { progressed on no more than two chemotherapies, } \\
\text { treated with afatinib } 50 \mathrm{mg} / \text { day monotherapy }\end{array}$ & $\begin{array}{l}\text { No ORR } \\
\text { PFS: } 54 \text { days for the HER } 2 \text { negative } \\
\text { and hormone receptor positive group; } \\
52 \text { days for the triple negative group AE: manageable }\end{array}$ \\
\hline Gunzer et $\mathrm{al}^{26}$ & 30 & $\begin{array}{l}\text { Phase 2: patients with stage IIIb or IV breast cancer } \\
\text { progressed on letrozole, treated with the combination } \\
\text { of letrozole plus afatinib }\end{array}$ & $\begin{array}{l}25 \% \text { SD (for more than } 16 \text { weeks) } \\
\text { AE: manageable }\end{array}$ \\
\hline
\end{tabular}

Abbreviations: PR, partial response; SD: stable disease; AE, adverse event; G, grade; ORR, overall response rate; PFS, progression free survival.

At the 32nd San Antonio Breast cancer Symposium in 2009, Harbeck and colleagues presented the results of a Phase II study of afatinib in patients with HER2-negative, hormone receptor-positive or triple negative metastatic breast cancer after no more than two prior chemotherapies. ${ }^{24}$ Among 56 enrolled patients, 50 were treated with afatinib $50 \mathrm{mg}$ once daily until disease progression.

Adverse events, mainly skin and gastrointestinal toxicities, were manageable. No objective responses were observed, but durable clinical benefit was observed in three patients with triple negative breast cancer (median duration of 184 days). Preliminary results indicated a median progression-free survival of 54 days for the HER2-negative, hormone receptor-positive patients and 52 days for the triplenegative patients.

At the recent 2012 ASCO meeting, Rimawi and colleagues presented results from a randomized open-label arm Phase II study comparing afatinib itself as a single agent versus trastuzumab and versus lapatinib in patients with HER2-positive, treatment-naïve, stage IIIA locally advanced breast cancer. ${ }^{25}$ After 6 weeks of treatment, afatinib showed a higher overall response rate compared to the other two agents, even if with a higher rate of AEs (especially gastrointestinal and skin toxicities).

Since the mechanism of acquired hormone-resistance may involve cross-talk between the ER and human epidermal growth factor receptor HER1/HER2 signaling pathways, Gunzer and colleagues evaluated the combination of afatinib plus letrozole. At the 2010 ASCO annual meeting, Gunzer and colleagues presented results from an open-label singlearm Phase II study with afatinib added to letrozole following disease progression on letrozole monotherapy. ${ }^{26}$ Primary endpoint was the rate of nonprogression at 16 weeks of therapy. A total of 28 mainly HER2-negative patients from 30 enrolled patients have been treated. The daily starting dose of afatinib was reduced from $50 \mathrm{mg}$ to $30 \mathrm{mg}$ due to skin and gastrointestinal toxicities (grade 3). Other reported AEs that were largely manageable were diarrhea, nausea, and asthenia. Preliminary data did not show any influence of afatinib on the pharmacokinetics of letrozole and vice versa. In this study, 7 of 28 patients evaluable were treated with stable disease for more than 16 weeks.

\section{Ongoing clinical trials}

There are several ongoing studies investigating afatinib in breast cancer patients, either in monotherapy or in association with other HER2-targeted therapies, hormonal drugs, or chemotherapeutic agents ${ }^{27}$ (Table 2).

Furthermore there are Phase II and III studies evaluating afatinib in combination with chemotherapy, in particular in association with vinorelbine (NCT01125566 - LUX Breast 1; NCT01325428; NCT01441596 - LUX-Breast 3) in patients with HER2-positive metastatic breast cancer after failure of trastuzumab or lapatinib treatment.

Recently, at the 2012 ASCO meeting, the Phase II LUXbreast 2 trial was presented. This trial will investigate the safety and efficacy of afatinib in HER2-positive metastatic breast cancer patients who progressed on prior trastuzumab and/or lapatinib. ${ }^{28}$

Moreover, afatinib is also being evaluated with another HER2-targeted therapy in an open-label, Phase I study (NCT00950742) to determine the MTD and assess safety in combination with trastuzumab in patients with HER2 and metastatic breast cancer.

Considering that about $40 \%-50 \%$ of HER2-positive tumors coexpress hormone receptors and this coexpression is often associated with reduced efficacy of endocrine therapy, afatinib was also tested in combination with hormonal therapy. The NCT00708214 study is a Phase I trial in patients with hormone refractory metastatic breast cancer where afatinib is administered in combination with letrozole. The aim of the study, which has just completed its accrual, is to 
Table 2 Main ongoing clinical trials

\begin{tabular}{|c|c|c|c|}
\hline Trial name (identifier) & Phase and status & Treatment scheme & Patient population \\
\hline $\begin{array}{l}\text { NCTOII } 25566 \\
\text { (LUX Breast I) }\end{array}$ & Phase 3: recruiting & $\begin{array}{l}\text { Vinorelbine }+ \text { trastuzumab vs } \\
\text { vinorelbine }+ \text { afatinib }\end{array}$ & I and II line metastatic breast cancer patients \\
\hline NCT0I325428 & Phase 2 : recruiting & $\begin{array}{l}\text { Afatinib alone or in combination } \\
\text { with vinorelbine }\end{array}$ & $\begin{array}{l}\text { HER } 2 \text { positive locally advanced or } \\
\text { metastatic inflammatory breast cancer }\end{array}$ \\
\hline $\begin{array}{l}\text { NCT0I44I596 } \\
\text { (LUX Breast 3) }\end{array}$ & Phase 2: recruiting & $\begin{array}{l}\text { Afatinib alone or in combination } \\
\text { with vinorelbine }\end{array}$ & $\begin{array}{l}\text { HER2 positive breast cancer with brain } \\
\text { metastasis after trastuzumab/ } \\
\text { lapatinib therapy }\end{array}$ \\
\hline NCT00826267 & Phase 2: completed & $\begin{array}{l}\text { Afatinib monotherapy vs } \\
\text { trastuzumab vs lapatinib }\end{array}$ & $\begin{array}{l}\text { HER2 positive treatment-naïve stage Illa } \\
\text { breast cancer patients (neoadjuvant setting) }\end{array}$ \\
\hline NCT007082। 4 & Phase 2: completed & $\begin{array}{l}\text { Afatinib in combination } \\
\text { with letrozole }\end{array}$ & $\begin{array}{l}\text { Hormone resistant metastatic } \\
\text { breast cancer patients }\end{array}$ \\
\hline NCT00425854 & Phase 2: completed & Afatinib in monotherapy & $\begin{array}{l}\text { HER2 negative metastatic breast cancer, } \\
\text { after maximum } 2 \text { lines of chemotherapy }\end{array}$ \\
\hline NCT00950742 & Phase I: active, not recruiting & $\begin{array}{l}\text { Afatinib in combination with } \\
\text { trastuzumab }\end{array}$ & $\begin{array}{l}\text { HER2 positive advanced refractory } \\
\text { breast cancer patients }\end{array}$ \\
\hline
\end{tabular}

assess progression-free rates after 16 weeks of treatment administration.

Finally, afatinib has also been tested in patients with HER2negative breast cancer: NCT00425854 is a recently completed open-label Phase II trial to assess the efficacy and safety of a once-daily oral dose of $50 \mathrm{mg}$ afatinib in two cohorts of patients with HER2-negative advanced breast cancer, after failure of no more than two chemotherapy regimens. NCT01531764 is a recruiting Phase II study evaluating safety and efficacy of afatinib in combination with vinorelbine in metastatic breast cancer patients pretreated with anthracycline and who have intermediate HER 2 status ( $2+$ by immunochemistry, but negative by fluorescence in-situ hybridization). NCT01531764 is estimated to be completed in 2016.

\section{Discussion and future perspectives}

Overexpression of HER2 predicts, in absence of treatment, a poor prognosis in breast cancer. Nowadays, different HER2-targeted therapies, such as trastuzumab and lapatinib, have significantly improved outcome in HER2-positive breast cancer patients. A further step forward has been made by dual targeting of HER2 with pertuzumab and trastuzumab in combination with chemotherapy. ${ }^{3}$ However, medical oncologists deal with patients progressing because of upfront or acquired drug resistance and, not rarely, by serious toxicities. An improved molecular understanding of breast cancer biology has led to the development of single-targeted and multitargeted agents for the treatment of breast cancer that has dramatically changed the approach to this disease. A number of strategies have been developed to deal with progressive HER2-positive metastatic breast cancer, which often maintains HER2 dependence.
Efficient blockade of HER2-dependent signaling may be achieved by simultaneous targeting in this biological cross-talk network of different HERs, as neratinib (HER1, HER2, and HER4) and afatinib (HER1 and HER2) do, reverting some of the possible mechanisms of resistance to trastuzumab. Both these agents have shown promising results in terms of response rates and progression-free survival in trastuzumab-exposed patients. ${ }^{6}$ However, treatment with neratinib was associated with several AEs such as diarrhea, nausea, vomiting, and fatigue, seen in the majority of patients (diarrhea grade 3-4 in 20\% of patients). Moreover, both agents have demonstrated a favorable activity compared with single-agent trastuzumab data. ${ }^{29}$ However, important points remain unsolved. The first important point related to the development of afatinib in breast cancer is the best clinical setting for use.

The experience with the reversible dual HER/EGFR tyrosine kinase inhibitor lapatinib, in patients with brain metastases from breast cancer ${ }^{30}$ suggests that small molecule inhibitors, differently from monoclonal antibodies, may act beyond the blood-brain barrier. In fact, it was shown that trastuzumab levels in cerebrospinal fluid are about 300fold lower than those in plasma, therefore indicating that trastuzumab cannot efficiently cross the blood-brain barrier. ${ }^{31}$ On the contrary, available data in mouse models suggest that due to the very low molecular weight $(<1 \mathrm{kDa}$ ) lapatinib can cross the blood-brain barrier easily. Similarly to lapatinib, afatinib could probably play a role in brain metastases. However, we are waiting for confirmation from the ongoing trials in this subset of patients treated with afatinib.

Second, similarly to what happens for other HER 2 inhibitors, resistance to afatinib is a frequent event. Important work 
needs to be done therefore to identify predictive factors of response and resistance beyond HER2 gene mutation and/or amplification. As shown for other HER2 inhibitors, primary and acquired resistance involves alterations in HER2 itself, coexpression or acquisition of bypass signaling through other receptors or intracellular signaling pathways, defects in mechanisms of cell cycle regulation or apoptosis, and host factors that may modulate drug response. A consistent number of neoadjuvant trials conducted in HER2-positive breast cancers treated with different anti HER2 agents (trastuzumab, pertuzumab, lapatinib) such as Neosphere, ${ }^{32}$ NeoALTTO $^{33-35}$ are currently validating predictors of response/resistance on tumor tissues such as transforming growth factor- $\alpha$, EGFR family members' activational status and phosphorylated downstream effectors.

Investigation of similar markers may predict also sensitivity to afatinib, therefore eliciting a more appropriate definition of a "molecular" setting predictive of benefit from treatment. In conclusion, it is likely that in the near future, afatinib will become part of our armamentarium for the treatment of HER2-overexpressing breast cancer patients. The challenge to clinicians will be how to use this drug at its best potential.

\section{Disclosure}

The authors report no conflicts of interest in this work.

\section{References}

1. Citri A, Yarden Y. EGF-ERBB signalling: towards the systems level. Nat Rev Mol Cell Biol. 2006;7:505-516.

2. Yarden Y, Sliwkowski MX. Untangling the ErbB signalling network. Nat Rev Mol Cell Biol. 2001;2:127-137.

3. Baselga J, Cortes J, Kim SB, et al. Pertuzumab plus trastuzumab plus docetaxel for metastatic breast cancer. N Engl J Med. 2012;366: 109-119.

4. Blackwell et al. Primary results from EMILIA, a phase III study of trastuzumab emtansine (T-DM1) versus capecitabine and lapatinib in HER2positive locally advanced or metastatic breast cancer previously treated with trastuzumab and a taxane. J Clin Oncol 30, 2012 (suppl; abstr LBA1).

5. Blackwell KL, Burstein HJ, Storniolo AM, et al. Randomized study of lapatinib alone or in combination with trastuzumab in women with ErbB2-positive, trastuzumab-refractory metastatic breast cancer. J Clin Oncol. 2010;28:1124-1130.

6. Burstein HJ, Sun Y, Dirix LY, et al. Neratinib, an irreversible ErbB receptor tyrosine kinase inhibitor, in patients with advanced ErbB2positive breast cancer. J Clin Oncol. 2010;28:1301-1307.

7. Geyer CE, Forster J, Lindquist D, et al. Lapatinib plus capecitabine for HER2-positive advanced breast cancer. $N$ Engl J Med. 2006;355: 2733-2743

8. Li D, Ambrogio L, Shimamura T, et al. BIBW2992, an irreversible EGFR/HER2 inhibitor highly effective in preclinical lung cancer models. Oncogene. 2008;27:4702-4711.

9. Miller et al. Phase IIB/III double-blind randomized trial of afatinib + best supportive care versus placebo + best supportive care in patients with NSCLC failing 1-2 lines of chemotherapy and erlotinib or gefitinib (LUX lung 1). Annals of Oncology 21 (Supplement 8).
10. Yamamoto $\mathrm{N}$ et al. A phase II trial of afatinib in patients with advanced non-small cell lung cancer previously treated with erlotib or gefitinib. J Clin Oncol 29: 2011 (suppl; abstr 7524).

11. Yang JC-H, Schuler MH, Yamamoto N, et al. LUX-Lung 3: A randomized, open-label, phase III study of afatinib versus pemetrexed and cisplatin as first-line treatment for patients with advanced adenocarcinoma of the lung harboring EGFR-activating mutations. ASCO Meeting Abstracts. 2012;30:LBA7500.

12. Minkovsky N, Berezov A. BIBW-2992, a dual receptor tyrosine kinase inhibitor for the treatment of solid tumors. Curr Opin Investig Drugs. 2008;9:1336-1346.

13. Eskens FA, Mom CH, Planting AS, et al. A phase I dose escalation study of BIBW 2992, an irreversible dual inhibitor of epidermal growth factor receptor 1 (EGFR) and 2 (HER2) tyrosine kinase in a 2-week on, 2-week off schedule in patients with advanced solid tumours. $\mathrm{Br} J$ Cancer. 2008;98:80-85.

14. Yap TA, Vidal L, Adam J, et al. Phase I trial of the irreversible EGFR and HER2 kinase inhibitor BIBW 2992 in patients with advanced solid tumors. J Clin Oncol. 2010;28:3965-3972.

15. Ang J, et al. A phase I study of daily BIBW 2992, an irreversible EGR7HER2 dual kinase inhibitor, in combination with weekly paclitaxel. J Clin Oncol 27, 2009 (suppl; abstr e14541).

16. Vermorken JB, Machiels JH, Rottey S. Phase Ib study evaluating the combintion of BIBW 2992 with two different standard chemotherapy regimens, cisplatin/paclitaxel (PT) and cisplatin/5FU (PF) in patients with advanced solid tumors $J$ Clin Oncol 28, 2010 (suppl; abstr e13521).

17. Hollebecque A et al. Phase I trial to assess the safety and pharmacokinetics of afatinib and weekly vinorelbine in patients with advanced solid tumors. J Clin Oncol 30, 2012 (suppl; abstr 3104).

18. Senellart $\mathrm{H}$, et al. Phase I safety and tolerability of once daily oral afatinib (A) in combination with docetaxel (D) in patients (pts) with relapsed or refractory advanced solid tumors.

19. Senellart H, et al. Phase I safety and tolerability of once daily oral afatinib (A) in combination with docetaxel (D) in patients (pts) with relapsed or refractory advanced solid tumors. J Clin Oncol 30, 2012 (suppl; abstr e13010).

20. Shaw H, Plummer R, Vidal L, et al. A phase I dose escalation study of BIBW 2992, an irreversible dual EGFR/HeR2 receptoor tyrosine Kinase inhibitor, in patients with advanced solid tumors. J Clin Oncol (Meeting Abstracts). 2006;24(18S):Suppl 3027.

21. Lewis N, Marshall J, Amelsberg A, et al. A phase I dose escalation study of BIBW 2992, an irreversible dual EGFR/HER2 receptor tyrosine kinase inhibitor, in a 3 week on 1 week off schedule in patients with advanced solid tumors. J Clin Oncol (Meeting Abstracts). 2006;24(18S): Suppl 3091.

22. Hickish T, et al. Use of BIBW2992, a novel irreversible EGFR/HER1 and HER2 tyrosine kinase inhibitor to treat patients with HER2-positive metastatic breast cancer after failure of treatment with trastuzumab. Cancer Res 2009;69(24 Suppl):Abstract nr 5060.

23. Lin NU, Winer EP, Wheatley D, et al. A phase II study of afatinib (BIBW 2992), an irreversible ErbB family blocker, in patients with HER2-positive metastatic breast cancer progressing after trastuzumab. Breast Cancer Res Treat. 2012;133(3):1057-1065.

24. Harbeck N, et al. BIBW2992, a novel irreversible EGFR/HER1 and HER2 tyrosine kinase inhibitor for the treatment of patients with HER2negative metastatic breast cancer after failure of no more than two prior chemotherapies. Cancer Res 2009;69(24 Suppl):Abstract nr 5062.

25. Rimawi MF, Aleixo SB, Alarcon Rozas A, et al. A neoadjuvant, randomized, open-label phase II trial of afatinib (A) versus trastuzumab (T) versus lapatinib (L) in patients (pts) with locally advanced HER2-positive breast cancer (BC). ASCO Meeting Abstracts. 2012; 30:606.

26. Gunzer K, et al. Addition of BIBW 2992, an irreversible inhibitor of EGFR/HER1 and HER2, to letrozole in estrogen receptor (ER)-positive metastatic breast cancer $(\mathrm{mBC})$ progressing on letrozole monotherapy. J Clin Oncol 28:15s, 2010 (suppl; abstr 1072). 
27. Data from www.clinicaltrial.gov (last update July 2012).

28. Hickish T, Tseng LM, Mehta AO, et al. LUX-breast 2: Phase II, openlabel study of oral afatinib in HER2-overexpressing metastatic breast cancer (MBC) patients (pts) who progressed on prior trastuzumab (T) and/or lapatanib (L). ASCO Meeting Abstracts. 2012;30:TPS651.

29. Ocana A, Amir E. Irreversible pan-ErbB tyrosine kinase inhibitors and breast cancer: current status and future directions. Cancer Treat Rev. 2009;35:685-691.

30. Lin NU, Dieras V, Paul D, et al. Multicenter phase II study of lapatinib in patients with brain metastases from HER2-positive breast cancer. Clin Cancer Res. 2009;15:1452-1459.

31. Pestalozzi BC, Brignoli S. Trastuzumab in CSF. J Clin Oncol. 2000;18: 2349-2351.

32. Gianni L, Pienkowski T, Im YH, et al. Efficacy and safety of neoadjuvant pertuzumab and trastuzumab in women with locally advanced, inflammatory, or early HER2-positive breast cancer (NeoSphere): a randomised multicentre, open-label, phase 2 trial. Lancet Oncol. $2012 ; 13: 25-32$.
33. Baselga J, Bradbury I, Eidtmann H, et al. Lapatinib with trastuzumab for HER2-positive early breast cancer (NeoALTTO): a randomised, open-label, multicentre, phase 3 trial. Lancet. 2012;379:633-640.

34. Chang JCN. TBCRC 006: a multicenter phase II study of neoadjuvant lapatinib and trastuzumab in patients with HER 2 overexpressing breast cancer. J Clin Oncol 29: 2011 (suppl; abstr 505).

35. Holmes F. A. Correlation of molecular effects and pathologic complete response to preoperative lapatinib and trastuzumab, separately and combined prior to neoadjuvant breast cancer chemotherapy. $J$ Clin Oncol 29: 2011 (suppl; abstr 506)

\section{Publish your work in this journal}

Breast Cancer: Targets and Therapy is an international, peerreviewed open access journal focusing on breast cancer research, identification of therapeutic targets and the optimal use of preventative and integrated treatment interventions to achieve improved outcomes, enhanced survival and quality of life for the cancer patient.

\section{Dovepress}

View the full aims and scopes of this journal here. The manuscript management system is completely online and includes a very quick and fair peer-review system, which is all easy to use. Visit http:// www.dovepress.com/testimonials.php to read real quotes from published authors.

Submit your manuscript here: http://www.dovepress.com/breast-cancer---targets-and-therapy-journal 